\title{
Factores impulsores de la propensión a innovar y la productividad en los sectores de servicios y la industria manufacturera en Colombia
}

DOI: https://doi.org/10.21158/01208160.n0.2020.2730

\section{Fecha de recepción: 02 de junio de 2020}

Fecha de aprobación: 25 de septiembre de 2020

Fecha de publicación: 30 de noviembre de 2020
Rafael García-Luna ${ }^{1}$ Universidad del Magdalena rgarcial@unimagdalena.edu.co

Diego Andrés Cardoso-López ${ }^{2}$ Universidad del Magdalena diegocardosolopez@gmail.com

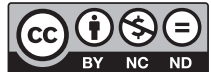

Cómo citar este artículo / To reference this article / Comment citer cet article / Para citar este artigo:

García-Luna, R.; Cardoso-López, D. A. (2020). Factores impulsores de la propensión a innovar y la productividad en los sectores de servicios y la industria manufacturera en Colombia. Revista Escuela de Administración de Negocios, Edición especial 2020, 33 - 52. DOI: https://doi.org/10.21158/01208160.n0.2020.2730

\section{Resumen}

El objetivo de este artículo es analizar los factores que impulsan la propensión a innovar y el desarrollo de la productividad en los sectores de servicios e industria manufacturera en Colombia -2013 a 2017-. Para su cumplimiento se utilizó una función de innovación con el fin de representar las características que inciden en la capacidad innovadora, en conjunto con vectores de características de las empresas y de insumos que permiten calcular el efecto de estos factores en la producción. Además, por medio de una función Cobb-Douglas se calculó la productividad total de los factores (PTF) y se examinó la variación de las productividades para ambos sectores. Entre los principales resultados se evidenció que el tamaño de las empresas, la inversión extranjera directa (IED) y la cooperación con otras instituciones tienen un impacto significativo en la propensión a innovar; así mismo, el porcentaje de capital humano y de exportaciones tienen un efecto positivo generalizado, el cual es mayor en el sector de servicios. En el cálculo de la productividad, el stock de capital físico, los trabajadores y las inversiones en investigación y desarrollo (I+D) tienen una participación positiva en el crecimiento empresarial de ambos sectores. Las variaciones de la productividad evidencian mayores incrementos y niveles más homogéneos del sector manufacturero en comparación con los servicios en los periodos estudiados.

Palabras claves: innovación: propensión a innovar; capacidad innovadora; desarrollo de productividad; investigación y desarrollo (I+D); sector de servicios; industria manufacturera.

\footnotetext{
${ }^{1}$ Doctor en Ciencias Sociales - Universidad del Norte. Magister en Ciencias económicas - Universidad Nacional de Colombia. Economista - Universidad del Magdalena. ORCID: https://orcid.org/0000-0001-7705-3571

${ }^{2}$ Economista - Universidad del Magdalena. ORCID: https://orcid.org/0000-0001-6767-0146
} 


\section{Drivers of the propensity to innovation and productivity in the service sectors and manufacturing industry in Colombia}

\section{Abstract}

The aim of this article is to analyze the factors that drive the propensity to innovation and productivity in the service and manufacturing sectors in Colombia from 2013 to 2017. In order to accomplish this goal, we used an innovation function to represent the characteristics that affect the innovative capacity of the company, together with vectors for the characteristics of the company and for inputs, that allow calculating the effect of these factors on the production. In addition, we calculated the total factor productivity (TFP) with a Cobb-Douglas function, and examined the variation in the productivity for both sectors. The main results make clear that the size of the companies, the foreign direct investment (FDI), and the cooperation with other institutions have a significant impact on the propensity to innovate; likewise, the percentage of human capital and exports has a widespread positive effect, which is greater in the service sector. In the calculation of productivity, the stock of physical capital, workers, and investment in research and development (R\&D) have a positive participation in the business growth of both sectors. Variations in productivity during the periods that were studied show greater increases and more homogeneous levels in the manufacturing sector than in the services sector.

Keywords: innovation: propensity to innovate; innovative capacity; productivity development; research and development (R\&D); service sector; manufacturing industry.

\section{$\longrightarrow \quad$ Fatores que impulsam a propensão para inovar e a produtividade nos setores de serviços e indústria manufatureira na Colômbia \\ Resumo}

O objetivo deste artigo é analisar os fatores que impulsionam a propensão para inovar e o desenvolvimento da produtividade nos setores de serviços e da indústria manufatureira na Colômbia -2013 a 2017-. Para o seu cumprimento, foi utilizada uma função de inovação para representar as características que influenciam a capacidade de inovação, juntamente com vetores de características das empresas e insumos que permitem calcular o efeito desses fatores na produção. Além disso, através de uma função Cobb-Douglas, calculou-se a produtividade total dos fatores (PTF) e examinou-se a variação das produtividades para os dois setores. Dentre os principais resultados, evidenciou-se que o porte das empresas, o investimento estrangeiro direto (IED) e a cooperação com outras instituições, têm impacto significativo na propensão a inovar; da mesma forma, o percentual de capital humano e de exportações têm um efeito positivo generalizado, que é maior no setor de serviços. No cálculo da produtividade, o estoque de capital físico, trabalhadores e investimentos em pesquisa e desenvolvimento $(\mathrm{P}+\mathrm{D})$ têm participação positiva no crescimento empresarial em ambos setores. As variações de produtividade mostram maiores aumentos e níveis mais homogêneos do setor manufatureiro em relação aos serviços nos períodos estudados.

Palavras-chave: inovação; propensão para inovar; capacidade inovadora; desenvolvimento da produtividade; pesquisa e desenvolvimento $(\mathrm{P}+\mathrm{D})$; setor de serviços; indústria manufatureira 
Facteurs de propension à l'innovation et à la productivité des secteurs des services et de l'industrie manufacturière en Colombie

\section{Résumé}

L'objectif de cet article est d'analyser les facteurs d'amélioration de la propension à l'innovation et au développement de la productivité dans les secteurs des services et de l'industrie manufacturière en Colombie sur la période 2013 - 2017. À cette fin une fonction d'innovation a été mise en place afin de représenter les paramètres influençant la capacité d'innovation en fonction des vecteurs caractéristiques des entreprises et des intrants qui permettent de calculer l'effet de ces facteurs sur la production. De plus, la productivité totale des facteurs (PTF) a été calculée au moyen d'une fonction Cobb-Douglas et la variation de la productivité de ces deux secteurs d’activité a été examinée. Parmi les principaux résultats mis en évidence, nous observons que la taille des entreprises, les investissements directs étrangers (IDE) et la coopération avec d'autres institutions ont un impact significatif sur la propension à innover. De même, le pourcentage en capital humain et les exportations semblent avoir un effet positif plus important dans le secteur des services. Concernant le calcul de la productivité, le stock de capital physique, les travailleurs et les investissements en recherche et développement (R\&D) participent positivement à la croissance des entreprises de ces secteurs. Enfin, les variations de productivité montrent des améliorations plus importantes et des niveaux plus homogènes dans le secteur manufacturier que dans celui des services sur les périodes étudiées.

Mots-clés: innovation; propension à innover; capacité d'innovation; développement de la productivité; recherche et développement $(\mathrm{R} \& \mathrm{D})$; secteur des services; industrie manufacturière. 


\section{Introducción}

Annolizar los factores determinantes de la con los distintos contextos empresariales es aún un reto, especialmente para países como Colombia. Distintas investigaciones convergen en que existe una relación positiva entre la inversión en investigación y desarrollo (I+D) con los resultados en productividad e innovación empresarial, lo cual evidencia cómo aquellos sectores que realicen más innovaciones en su cadena de producción dinamizarán el crecimiento de la productividad; pero las diferencias en las capacidades y las características de las empresas, aun en un mismo sector, hacen más complejo el análisis (Crespi, Tacsir y Vargas, 2016; Morikawa, 2019).

Reconociendo las complejidades y la necesidad de abordar el problema de la relación entre innovación y productividad en economías en desarrollo, el objetivo de este artículo es analizar los factores que impulsan la propensión a innovar y el desarrollo de la productividad de las empresas del sector de servicios e industria manufacturera en Colombia, para lo cual se estima, en primera instancia, un modelo Probit a fin de calcular la probabilidad de innovar de las firmas pertenecientes a los mencionados sectores.

Se utilizará una función estilo Cobb-Douglas para analizar la función de producción y el residuo de Solow, estimándola bajo la metodología de factibles mínimos cuadrados generalizados (FMCG), con base en los datos de la Encuesta de Desarrollo e Innovación Tecnológica de los Sectores de Servicios y Comercio EDITS $^{3}$ —versiones V y VI, periodos 2014-2015 y 2016-2017—, y la EDIT manufacturera —versiones VII y VIII, periodos 2013-2014 y 2015-2016-.
A fin de cumplir el objetivo planteado, el presente artículo se organiza en cuatro partes. La primera, seguida de esta introducción, establece una revisión de literatura; en la segunda se presentan los datos y la metodología. En la tercera se despliegan los resultados y en la cuarta se establecen las principales reflexiones con respecto a lo analizado durante el proceso investigativo.

\subsection{Productividad e innovación: una relación de ida y vuelta}

La productividad, en términos generales, puede definirse como el grado máximo de eficiencia alcanzada en la producción con base en la utilización de factores, cuantificando el empleo de insumos de manera eficaz con el fin de obtener una unidad de un bien (Svyerson, 2011). A su vez, la innovación se entiende como un conjunto de actividades o sus resultados, de las que se obtienen productos o procesos nuevos o mejorados - o una combinación de ambos- que están dispuestos para el mercado o la empresa (OECD y Eurostat, 2018 $)$; de manera que es un elemento sustantivo para el crecimiento económico, dado que innovar genera ventajas comparativas y es la relación que existe entre innovación y productividad la que puede explicar las diferencias de desarrollo entre empresas o países (Raymond y Louis St-Pierre, 2010).

Es posible indicar que la relación entre innovación y productividad es positiva, así como que la selección de procesos innovadores no aleatorios impulsa el crecimiento de la productividad entre las firmas (Mañéz, Rochina-Barrachina, Sanchis y

\footnotetext{
${ }^{3}$ Las encuestas las realizó el Departamento de Administrativo Nacional de Estadística de la República de Colombia (DANE).

${ }^{4}$ Oficina de Estadística para las Comunidades Europeas (EurostaT) y la Organización para la Cooperación y Desarrollo Económicos (OCDE).
} 
Sanchis, 2013); en este sentido, para el caso de seis economías latinoamericanas, Crespi y Zuniga (2012) demostraron que la probabilidad de aumentar la productividad con base en la inversión en I+D es alta y genera una fuerte expansión de los niveles alcanzados de productividad por trabajador en la mayoría de las economías estudiadas.

La relación entre innovación y productividad explica, entonces, la movilización de capital y trabajo a la incorporación de procesos de $\mathrm{I}+\mathrm{D}$, generando actividades con mayor uso de conocimiento e incrementos de la productividad sectorial y de las empresas (Carvalho y Avellar, 2017; Lee, Lee y Garrett, 2017). Cuando las firmas alcanzan en su estructura de producción un grado máximo de actividad, se generan efectos spillover, estimulando la existencia de aumentos en la productividad del grupo de empresas que están consolidando procesos más eficientes (Heggedal, Moen y Preugschat, 2017).

En el análisis de la relación entre innovación y productividad se han evidenciado algunas relaciones de ida y vuelta, es decir, la innovación impacta la productividad y viceversa (véase la Figura 1). No obstante, es perentorio aclarar que las innovaciones inciden en cierta medida en el crecimiento de la productividad, pero no describen por completo los cambios en esta, la cual también se ve impactada por elementos del ecosistema o entorno productivo.

Figura 1. Relaciones entre factores de la innovación y productividad

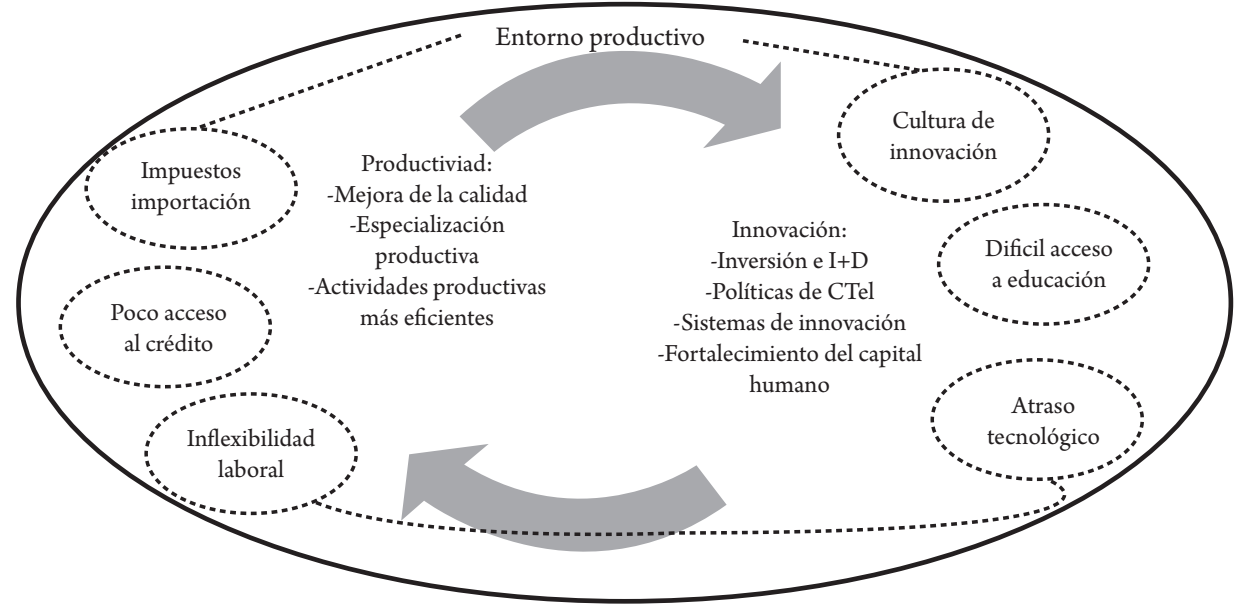

Fuente. Elaboración propia.

Los procesos causales entre innovación y productividad también pueden explicarse por factores internos, tales como las inversiones en $\mathrm{I}+\mathrm{D}$, las patentes y los sistemas de captura de información de las firmas, los cuales son elementos que incrementan la productividad a largo plazo; así mismo, por algunos factores externos como la implementación de políticas para el impulso de la ciencia, tecnología e innovación (CTI) que buscan mejorar el desarrollo de innovaciones, que impactan positivamente el crecimiento de la productividad (Sánchez-Sellero, Sánchez-Sellero, Sánchez-Sellero y Cruz-González, 2014; Tello, 2017).
Los efectos positivos de la innovación sobre la productividad se han evidenciado en muchos casos, tal como lo determinaron Villarreal, Lucio-Arias, Albis y Mora (2014), quienes por medio de métodos estadísticos analizaron los efectos de los procesos innovadores y demostraron que son positivos sobre la productividad al ser, en su mayoría, liderados por empresas pequeñas. Es fundamental aclarar que existen otros factores relacionados con el entorno productivo (véase la Figura 1) que pueden afectar la dinámica empresarial y el desarrollo de mudanzas estructurales hacia actividades con más altos niveles de innovación y productividad para empresas y 
sectores (Nakamura, Kaihatsu y Yagi, 2019; Ramírez, Zubieta y Bedoya, 2014).

El cambio hacia actividades con mayor productividad requiere la incorporación de nuevos e innovadores esquemas de producción y organización que permitan una mejor adaptación a los nuevos objetivos productivos (Cainelli, Evangelista y Savona, 2006). Se puede establecer, entonces, que firmas con baja capacidad instalada en términos de inversiones en capital o trabajos dedicados a la realización de innovaciones obtendrán tasas de retorno en productividad más bajas (Haskel y Wallis, 2013); es necesario el fortalecimiento de la capacidad de innovación de las empresas y la generación de derrames de conocimiento que puedan mejorar la capacidad instalada de producción que tenga un país, optimizando su capacidad productiva, tal como se logró para el caso de China (Brondino, 2019).

\subsection{Factores que impactan la productividad industrial y de los servicios}

Diversos factores impactan la productividad del sector industrial, entre los que se pueden destacar las innovaciones en la estructura de producción, las mejoras en la escolaridad, la especialización, el avance en la apertura comercial, las reformas políticas que promocionan la competitividad, los sistemas de calidad y la eficiencia en los mercados, aspectos todos que mejoran la productividad y establecen el sector industrial como un actor importante en el crecimiento de las economías (Broadberry, 1995; Fleury, 1995; Varella y Cabral, 2009). En relación con las reformas políticas y sus efectos, para el caso de la economía China la transformación del modelo económico ha generado la creación de cadenas de especialización e incrementos de los niveles potenciales de la PTF (Chen, Jefferson y Zhang, 2011).

La innovación se considera uno de los factores que impulsan la productividad del sector industrial, al igual que otros elementos tales como las facilidades para la importación de nuevos bienes de capital, el aumento de la competencia y las mejoras de la infraestructura logística. Sin embargo, algunos aspectos disminuyen la dinámica de la productividad, como, por ejemplo, las rigideces del mercado laboral, los altos costos laborales, el bajo acceso al financiamiento y los impuestos a materias primas importadas que impactan especialmente a las empresas de menor tamaño (Montes-Rojas y Santamaría, 2007; Singh, Gupta y Juneja, 2018).

Para el caso del sector servicios la innovación también es un elemento fundamental en el propósito de explicar su productividad, y esta es resultado, entre otros aspectos, de la rápida movilización de conocimiento interno, además de la interacción de sistemas de innovación en servicios (Weinstein y Gallouj, 1997). Los procesos de innovación en el sector servicios requieren capacidades internas de las empresas y de aprendizaje de los mercados por lo que se hace complejo innovar-, al ser, en su mayoría, las firmas que trabajan en el uso intensivo de herramientas tecnológicas las que generan servicios innovadores y con alto valor agregado (Weerawardena y Mccoll-Kennedy, 2002; Zagler, 2002).

Las empresas de servicios que realizan un proceso de adopción y transformación creativa de tecnologías y acumulación de capital físico aumentan el crecimiento de sus rendimientos marginales, por lo que las tecnologías envueltas en los servicios deben ser flexibles en su modo de uso para que generen una futura expansión de la innovación (Chapman, Soosay y Kandampully, 2002; Ozyilmaz, 2001). En el caso de las economías pertenecientes a la OECD, su expansión económica fue potencialmente impulsada por los crecimientos de redes de conocimiento, del capital humano e inversiones en capital fijo en el sector de los servicios, elementos que ampliaron la frontera de utilización de factores y la generación de innovación (Vang y Zellner, 2005).

La promoción de la innovación en los servicios impacta de manera positiva la productividad, por lo que Junarsin (2010) propone cinco fases a fin de fomentarla: a) la planeación de ideas relevantes para el mercado objetivo, b) el diseño de instrumentos, c) la implementación, d) los indicadores de 
funcionamiento, ye) las recomendaciones de política productiva. Otros elementos fundamentales para la generación de innovación en los servicios son las redes productivas y la configuración de interfaces de conocimientos sobre articulación de producción en la economía (Agarwal y Selen, 2011).

En la dinámica de los servicios, el acervo de capital humano en términos de activo en la cadena de valor es muy importante, tal como lo visualizan Pasban y Nojedeh (2016), quienes explican que este elemento es clave para el crecimiento económico y para mejorar las habilidades de la población. Mestieri, Schauer y Townsend (2017) afirman que se deben asegurar ciertos factores como la escolaridad de los niños, bajos costes de entrada a los mercados locales e internacionales y la consolidación de condiciones efectivas para el sector productivo, principalmente, en sectores intensivos en mano de obra como lo son los servicios.

Ahora bien, con la combinación de sistemas tecnológicos, de capital humano especializado y de sectores altamente productivos -industria o servicios- se crea una dinámica productiva positiva en las firmas (Grönroos y Ojasalo, 2015); en la industria, el consumo de tecnologías de la información y la comunicación es muy importante para la innovación y la productividad, aunque el sector de servicios muestra mayores avances en su utilización, por lo que el sector manufacturero debe enfocar sus inversiones en mejorar las innovaciones especialmente las tecnológicas (Aboal y Tacsir, 2018).

La innovación en servicios puede entenderse entonces como la implantación de ideas que crean soluciones con bienes inmateriales, sin necesidad de acelerar iniciativas de producción industrial y creando un ambiente innovador entre oferente y consumidor (Machado y Tello-Gamarra, 2017). Sin embargo, es necesario entender que, además de la innovación, los factores que afectan la productividad en los servicios en comparación con la industria manufacturera son diferentes, tal como lo planteó Rath (2018) para el caso de la India, en el que evidenció que solo la intensidad del capital afecta la productividad tanto de empresas de servicios como industriales.

\section{Metodología}

$\mathrm{E}^{\mathrm{s}}$ este artículo se asume que la innovación mejora la utilización de los factores productivos, por lo que impacta de forma positiva la productividad, y esta, a su vez, mantendrá el desarrollo de innovaciones. Aunque para este caso se analiza la influencia que ejercen distintos factores en la propensión a innovar y en la productividad de empresas pertenecientes a los sectores de servicios e industria manufacturera de Colombia, sin el análisis de causalidades de doble vía -innovación-productividad-innovación-.

En primera instancia, se estiman modelos empíricos para comprender el comportamiento de la propensión a innovar de las firmas y la intensidad de la innovación por parte de estas. Finalmente, se desarrollan funciones de producción con el fin de examinar la productividad estimada de los sectores de servicios y manufacturas, determinando los sectores proclives a tener productividades más homogéneas, teniendo en cuenta sus capacidades de generar innovaciones.

\subsection{Datos y periodo de análisis}

La base de datos a utilizar es la Encuesta de Desarrollo e Innovación Tecnológica (EDIT), desarrollada por el Departamento Administrativo Nacional de Estadísticas de Colombia (DANE), en sus aplicaciones para servicios e industria manufacturera; se utilizan las versiones Vy VI de la EDIT aplicadas al sector de servicios que comprende, respectivamente, los periodos 2014-2015 y 2016-2017; para analizar la industria manufacturera se usan las versiones VII y VIII referentes a los periodos de 2013-2014 y 20152016. 
Se utilizan las definiciones de tipos de empresas innovadoras ${ }^{5}$ establecidas en la EDIT:

- innovadoras en sentido estricto se definen las empresas que en el periodo de referencia obtuvieron al menos una innovación en un bien o servicio para el mercado internacional;

- innovadoras en sentido amplio son las empresas que durante el periodo de estudio tuvieron al menos una innovación en un bien o servicio en el mercado nacional, o que implementaron nuevas líneas de producción;

- potencialmente innovadoras se definen las empresas que en el periodo de referencia no registraron ninguna innovación, pero informan que realizaron algún proceso innovador que no culminaron. Por el contrario, las firmas catalogadas como no innovadoras son aquellas que en el periodo de referencia no realizaron ningún tipo de innovación (DANE, 2017).

Para la construcción de la base de datos las empresas catalogadas como no innovadoras no harán parte de este análisis, debido a la ausencia de información sobre sus características empresariales que permita estimar la propensión a innovar. Se escogerán las empresas innovadoras tanto en sentido estricto como amplio y potenciales para el sector servicios, del cual se analizarán 2122 firmas en el periodo 2014-2015 y 2155 para 2016-2017. En el caso de la industria manufacturera, en el periodo 2013-2014 se analizarán 2248 firmas innovadoras y 2254 para 2015-2016. Las estimaciones se realizarán utilizando el software Stata (Software for Statistics and Data Science) versión 16.

\subsection{Estimación de la propensión a innovar}

A fin de estimar la propensión a innovar se considera el enfoque de los modelos de dos etapas - modelo de Heckman-, con base a una serie de características de los insumos y la relación de estos factores en una función de innovación, tomando como referencia los trabajos de Heckman (1976), Hall, Lotti y Mairesse (2009), así como de Kriaa y Karray (2010).

A partir de este modelo, se construyen dos ecuaciones: en la primera se calcula la propensión de las empresas a realizar innovaciones con base en una serie de insumos; además, dichas innovaciones se representan por una variable categórica proxy que revela información cualitativa sobre el monto de inversión para actividades de $\mathrm{I}+\mathrm{D}$ y se calcula el efecto cuantitativo de los insumos sobre los niveles de innovación, es decir, sobre los niveles de I+D.

En la primera etapa se estima un modelo probit para calcular la probabilidad de innovar de las empresas; en esta situación, la decisión de innovar está explicada por las inversiones en $\mathrm{I}+\mathrm{D}$, por tanto, al ser un modelo de variable dependiente limitada, la decisión se basa en los niveles de inversión, en el que un monto superior a cero significa que la empresa innova tomando valores de 1-; en caso contrario, este será 0 . Por otra parte, las variables independientes son un vector de características empresariales .

Del mismo modo, es necesario especificar que este vector de características empresariales es un conjunto de subvectores de variables independientes que recogen aspectos relacionados con las empresas, identificación de dificultades técnicas y características relacionadas con la capacidad instalada en términos de exportación y capital humano (estas variables están reflejadas en la Tabla 2). Así, se ajusta el proceso de selección de firmas u observaciones.

\footnotetext{
${ }^{5}$ Definición de firmas innovadoras de acuerdo con la propuesto en la Encuesta de Innovación y Desarrollo Tecnológico EDIT que es realizada por el DANE para la industria manufacturera y para los sectores de servicios y comercio.
} 
La segunda ecuación intenta modelar los efectos de las variables independientes que corresponden al vector de características de las firmas sobre el logaritmo de la inversión en I+D de las empresas del sector de servicios y manufacturas. Esta estimación tiene como objetivo identificar el efecto de las variables sobre la intensidad de la inversión y de corregir el sesgo de selección de las empresas. Además, en esta segunda ecuación se encuentra implícita la razón de Mills como medida de corrección de selectividad; esta medición se deriva del proceso de estimación del análisis de probabilidad. En consecuencia, la representación econométrica del modelo Heckman, aplicado a los procesos de innovación, se representa de la siguiente forma:

$H=\left\{\begin{array}{ll}I_{i t}=\beta X_{i t}+\varepsilon_{i t} I_{i t}=1 \text { si } I+D>0 \text { ó } & I_{i t}=0 \text { si } I+D=0 \\ \ln I^{i t}=\beta X_{i t}+\varphi \lambda_{i t}+\varepsilon_{i t} & \text { (1) }\end{array}\right\}$

La estimación de ambas ecuaciones permite, en primera instancia, identificar aquellos factores que son necesarios en el proceso de innovación en la empresa. De igual forma, concede una perspectiva de la dirección del efecto sobre las inversiones en $\mathrm{I}+\mathrm{D}$ como instrumento de las innovaciones, para lo cual se analizan los efectos marginales de las variables independientes sobre las variables dependientes. Finalmente, en la primera etapa de este proceso se intenta clasificar las empresas que tienen una propensión mayor a generar innovaciones $y$, en la segunda, se identifica la dirección del impacto en la innovación tanto en procesos como en producto, especificando un sesgo de selección entre especificidades en las firmas.

\subsection{Modelo para la estimación de la productividad}

La estimación de productividad de empresas o regiones requiere la especificación de funciones de producción. Para esto, Van Biesebroeck (2004), Svyerson (2011), Beveren (2012), Messa (2014) y Camino-Mogro (2017) cimentaron un enfoque de representación de la economía con una función de producción de estilo Cobb-Douglas. Con base en estos estudios, se pretende estimar la productividad de las firmas colombianas del sector de servicios e industria manufacturera en cada periodo establecido de la siguiente forma:

$$
Y_{i t}=A_{i t} K_{i t}^{a} R_{i t}^{\beta} L_{i t}^{\delta *} e^{X_{i t}}
$$

En donde el termino $Y_{i t}$ es el ingreso corriente de las firmas en un tiempo determinado; $A_{i t}$ es la medida de progreso técnico de Hicks-Neutral y se considera un medio para estimar la productividad total de los factores; $K_{i t}$ se refiere al stock de capital físico en actividades de CTI, excluyendo el monto en $I+D ; R_{i t}$ es el monto de inversión en $I+D ; L_{i t}$ representa la cantidad de empleados de las empresas; $y e^{X_{i t}}$ es un vector de variables exógenas que acumulan características extensivas de la empresa - este vector se utiliza durante el proceso de estimación de la propensión a innovar-.

Ahora bien, se procede a linealizar la función de producción por medio de la aplicación de logaritmos naturales. Además, los términos conformados por letras griegas son las elasticidades de uso de insumos y la suma de los parámetros en la estimativa representan sus rendimientos a escala.

$$
\begin{gathered}
y_{i t}=a_{i t}+\theta X_{i t}+a k_{i t}+\beta r_{i t}+\delta l_{i t}+\varepsilon_{i t} \\
\ln A_{i t}=a_{i t}+\varepsilon_{i t}
\end{gathered}
$$

En el caso de $a_{i}+\varepsilon_{i}$, el primero es la media de la productividad total de los factores de las empresas y el segundo significa la desviación estándar de las firmas. La desviación estándar puede hallarse al despejar la función de producción linealizada con el fin de obtener los criterios de desviación de productividad de las empresas arrojando las mediciones de productividad total de los factores (PTF).

$$
\varepsilon_{\mathrm{it}}=\mathrm{y}_{\mathrm{it}}-\mathrm{a}_{\mathrm{it}}-\theta \mathrm{X}_{\mathrm{it}}-\alpha \mathrm{k}_{\mathrm{it}}-\beta \mathrm{r}_{\mathrm{it}}-\delta \mathrm{l}_{\mathrm{it}}
$$


Por consiguiente, los parámetros pueden estimarse por medio de la metodología de mínimos cuadrados ordinarios (MCO). No obstante, la estimación de una función de producción con este método posee ciertas limitaciones metodológicas que pueden generar estimaciones inconsistentes, pero, dadas las características presentes en la composición de los datos, es la metodología que mejor se ajusta. A pesar de que las estimaciones de funciones de producción por medio de $\mathrm{MCO}$ fueron las primeras estimaciones que plantaron una base en la concepción de la productividad, también presentan limitaciones en la especificación de parámetros (Camino-Mogro, 2017; Eslava, Haltiwanger, Kugler, y Kugler, 2013; Levinsohn y Petrin, 2003; Petrin, Poi y Levinsohn, 2004).

En relación con las limitaciones, estos autores concilian en que los problemas radican en la correlación entre los mismos insumos y la productividad estimada, violándose en principio la independencia entre parámetros. Adicionalmente, el incumplimiento del anterior principio generaría que la variable que representa la dinámica de productividad no cumpla con el criterio de insesgadez, no representando las mejores estimaciones de productividad. En este propósito, plantean metodologías como MCO en diferencias, hasta modelos generalizados de momentos (MGM), para solucionar estos inconvenientes que posee la estimación por MCO (Camino-Mogro, 2017; Chung, 2018; Dimelis y Papaioannou, 2011; Svyerson, 2011). Así, una de las estrategias a usar es la estimación de factibles mínimos cuadrados generalizados (FMCG) para la estimación de elasticidades consistentes e insesgadas.
Ahora, con base en la especificación realizada por Subramanian, Anderson y Lee (2005), se establece un marco en el cual sea posible cuantificar las mediciones de productividad, usando FMCG con el objetivo de obtener estimaciones consistentes y que la medición de productividad no esté relacionada con el resto de insumos o características generales de las empresas, cumpliéndose el criterio de exogeneidad. Así mismo, es necesario que las estimaciones de los residuos del modelo se descompongan en dos subresiduos, uno en el que presente la medida de error para todas las firmas en un año indicado y otro que es identificado como la heterogeneidad no observada. De esta forma, las estimaciones por FMCG alcanzan este enfoque y permiten que las estimaciones sean consistentes.

Es importante mencionar que, en razón a la estructura transversal de los datos recopilados por la encuesta, las estimaciones por medio de esta estrategia permiten la mejor especificación posible. Igualmente, se reconocen otros métodos que beneficiarían este análisis, pero por el uso de datos de empresas no identificadas en el tiempo resultaría complejo el uso de otras estrategias.

Finalmente, se analiza la propensión a innovar por parte de las firmas utilizando el enfoque de Heckman para corregir sesgos de selección y se examinan los diferentes caracteres que confieren credibilidad a las variables, así como su relevancia dentro de la producción de las firmas innovadoras. Esto genera, por medio de análisis de gráficos de densidad y análisis de constantes, la medida de productividad, la heterogeneidad o la homogeneidad del mercado con respecto a la productividad generada en las firmas. 


\section{Resultados}

\subsection{Propensión a innovar en servicios e industria manufacturera}

$\mathrm{Al}$ analizar la composición de las empresas estudiadas en las EDIT utilizadas se evidencia que entre un $70 \%$ y un $75 \%$ de las empresas no generan innovaciones (véase la Tabla 1). Las empresas innovadoras en sentido estricto y amplio se agruparán en la categoría de innovación definida, mientras las innovadoras potenciales como innovación no definida.

Tabla 1. Distribución de las firmas encuestadas con relación a la realización de innovaciones

\begin{tabular}{|c|c|c|c|c|}
\hline Tipo de empresas & EDITS V & EDITS VI & EDITM VII & EDITM VIII \\
\hline $\begin{array}{c}\text { Innovadoras en sentido estricto y amplio - } \\
\text { innovación definida- }\end{array}$ & $18,04 \%$ & $19,11 \%$ & $19,35 \%$ & $21,67 \%$ \\
\hline Innovadoras potenciales -innovación no definida- & $8,32 \%$ & $5,80 \%$ & $6,11 \%$ & $6,69 \%$ \\
\hline No innovadoras & $73,65 \%$ & $75,09 \%$ & $74,53 \%$ & $71,64 \%$ \\
\hline
\end{tabular}

Fuente. Elaboración propia con base en DANE, 2017.

En el proceso de identificación de variables que determinan la propensión a innovar se escogieron las que, de acuerdo con la literatura, impactan la realización de innovaciones, utilizando los datos de las empresas con innovación definida —innovadoras en sentido estricto y amplio- y con innovación no definida - innovadoras potenciales o con intenciones de innovar- (véase la Tabla 2); además, estas variables se relacionan con recursos y capacidades que impactan la función de producción y pueden describir los niveles de productividad agregados y de las empresas (Hussinger, 2008; Kriaa y Karray, 2010; Villarreal, Lucio-Arias, Albis y Mora, 2014).

Tabla 2. Caracterización de variables utilizadas en el cálculo de la propensión a innovar

\begin{tabular}{|l|l|c|}
\hline \multicolumn{1}{|c|}{ Variable } & \multicolumn{1}{c|}{ Especificación de las variables } & Tipo \\
\hline Log de capital en I+D & Logaritmo natural de las inversiones en I+D & Logarítmica \\
\hline Capital de I+D & 1 si la firma invierte en actividades de I+D, si no 0 & Dicotómica \\
\hline Tamaño de la empresa & 1 si la firma es grande a nivel de trabajadores, si no 0 & Dicotómica \\
\hline Inversión extranjera directa (IED) & 1 si la firma posee IED mayor al 10\%, sino 0 & Dicotómica \\
\hline Cooperación & $\begin{array}{l}1 \text { si la firma coopera con otras instituciones estatales } \\
\text { o no estatales, si no 0 }\end{array}$ & Dicotómica \\
\hline Falta de personal cualificado & 1 si la firma conoce sus obstáculos, si no 0 & Dicotómica \\
\hline Falta de protección de propiedad intelectual & 1 si la firma conoce sus obstáculos, si no 0 & Dicotómica \\
\hline Falta de protección a la imitación & 1 si la firma conoce sus obstáculos, si no 0 & Dicotómica \\
\hline Escases de recursos propios & 1 si la firma conoce sus obstáculos, si no 0 & Dicotómica \\
\hline Escases de información del mercado & 1 si la firma conoce sus obstáculos, si no 0 & Dicotómica \\
\hline Exportaciones —\%— & Porcentaje de exportaciones sobre ingresos & Porcentaje \\
\hline Capital humano —\%— & $\begin{array}{l}\text { Porcentaje de capital humano sobre el total de } \\
\text { trabajadores }\end{array}$ & Porcentaje \\
\hline
\end{tabular}

Fuente. Elaboración propia con base en DANE, 2017. 
Para la estimación del modelo Heckman de dos etapas se ha seleccionado como variable dependiente, en la primera ecuación, la propensión a invertir en actividades de $\mathrm{I}+\mathrm{D}$, dado que es una variable categórica. Por otra parte, como variable dependiente de la segunda ecuación se identifica el logaritmo natural de la inversión de $\mathrm{I}+\mathrm{D}$, denominado como la intensidad en $\mathrm{I}+\mathrm{D}$.

Estimando la primera etapa del modelo y examinando sus efectos marginales (véase la Tabla 3) se identifica que, a través de los sectores económicos, un tamaño mayor de la empresa —en número de empleadosgenera un efecto positivo en la decisión de innovar; igualmente, el nivel de cooperación de las firmas con instituciones gubernamentales y no gubernamentales genera un ambiente positivo en las empresas para incrementar los niveles de innovación tanto en el sector de servicios como en las manufacturas. El capital extranjero indicó una disminución de la probabilidad de innovar, situación que puede ser explicada como un efecto condicional del capital extranjero para inversión, en el cual las innovaciones se producen en otros contextos y solo se asimilan en el país receptor (Veugelers, 1997).

En relación con los principales obstáculos para la innovación, la falta de personal cualificado para la generación de innovaciones presenta múltiples efectos tanto en el sector de servicios como en el manufacturero, de manera que es positivo $y$ significativo para el primer periodo del sector de servicios -2014-2015-. En ambos sectores se evidenció que la protección de la propiedad intelectual tiene un efecto positivo sobre la propensión marginal a innovar de las empresas.
Al analizar el resto de barreras como la falta de protección a la imitación, la escasez de recursos propios o de información del mercado objetivo, se establece que el reconocimiento de estas dificultades estimula la innovación por medio de políticas de promoción dentro de la firma (Kriaa y Karray, 2010). El porcentaje de exportaciones sobre los ingresos totales de la firma tiene un impacto positivo en la intensidad de la generación de innovación reflejada en la decisión de invertir en $\mathrm{I}+\mathrm{D}$, en mayor medida en el sector manufacturero que en los servicios fenómeno que se explica por el efecto de la mayor transabilidad de los productos manufactureros frente a los servicios-.

La conformación del capital humano es positivo y significativo en la mayoría de los periodos con relación a la probabilidad de innovar, lo que afianza la relación directa entre el stock de conocimiento y los niveles de innovación (Halpern y Muraközy, 2012; Kleinknecht, 1996; Klomp y Leeuwen, 2001).

En la columna 2 de la tabla 3 se describen los coeficientes correspondientes a la segunda etapa para el sector de servicios y manufactura. Se halla que, para ambos periodos del sector manufacturero, el vector de variables independientes es significativo; no obstante, la percepción de la dificultad presentada por las firmas relacionada con la falta de personal cualificado no es significativa. Igualmente, en el primer periodo de la encuesta en el sector de servicios se reconoce el papel significativo del conjunto de variables independientes sobre la intensidad de la inversión en $\mathrm{I}+\mathrm{D}$, sin embargo, el reconocimiento de barreras tales como la falta de propiedad intelectual y el porcentaje de exportaciones tienden a no cobrar significancia en el modelo. 
Factores impulsores de la propensión a innovar y la productividad en los sectores de servicios y la industria manufacturera en Colombia

Tabla 3. Estimaciones de la propensión a innovar para el sector manufacturero y de servicios — modelo de Heckman-

\begin{tabular}{|c|c|c|c|c|c|c|c|c|}
\hline & \multicolumn{2}{|c|}{ EDITM 2013-2014 } & \multicolumn{2}{|c|}{ EDITM 2015-2016 } & \multicolumn{2}{|c|}{ EDITS 2014-2015 } & \multicolumn{2}{|c|}{ EDITS 2016-2017 } \\
\hline & $-1-$ & $-2-$ & $-1-$ & $-2-$ & $-1-$ & $-2-$ & $-1-$ & $-2-$ \\
\hline \multirow{2}{*}{$\begin{array}{l}\text { Tamaño de la } \\
\text { empresa }\end{array}$} & $0,175^{* * *}$ & $11,35^{* * *}$ & $0,206^{* * *}$ & $10,24^{* * *}$ & $0,115^{* * *}$ & $7,779^{* * *}$ & $0,134^{* * *}$ & $10,57^{* * *}$ \\
\hline & $-0,022-$ & $-1,532-$ & $-0,024-$ & $-1,255-$ & $-0,017-$ & $-0,730-$ & $-0,021-$ & $-2,143-$ \\
\hline \multirow[t]{2}{*}{ IED } & $-0,0478^{* *}$ & $-4,970^{* * *}$ & $-0,0494^{* *}$ & $-5,010^{* * *}$ & $-0,287^{* * *}$ & $-19,19^{* * *}$ & $-0,0626^{* * *}$ & $-7,868^{* * *}$ \\
\hline & $-0,020-$ & $-0,466-$ & $-0,021-$ & $-0,366-$ & $-0,020-$ & $-1,866-$ & $-0,022-$ & $-1,027-$ \\
\hline \multirow[t]{2}{*}{ Cooperación } & $0,113^{* * *}$ & $6,940^{* * *}$ & $0,137^{* * *}$ & $6,261^{* * *}$ & $0,135^{* * *}$ & $9,033^{* * *}$ & $0,162^{* * *}$ & $12,30^{* * *}$ \\
\hline & $-0,020-$ & $-1,054-$ & $-0,020-$ & $-0,897-$ & $-0,017-$ & $-0,832-$ & $-0,021-$ & $-2,562-$ \\
\hline \multirow{2}{*}{$\begin{array}{l}\text { Falta de personal } \\
\text { cualificado }\end{array}$} & 0,00589 & 0,132 & $-0,00253$ & 0,0104 & $0,0442^{* *}$ & $2,705^{* * *}$ & $-0,0125$ & $-0,871^{* * *}$ \\
\hline & $-0,023-$ & $-0,203-$ & $-0,023-$ & $-0,204-$ & $-0,020-$ & $-0,367-$ & $-0,025-$ & $-0,322-$ \\
\hline \multirow{2}{*}{$\begin{array}{l}\text { Falta de } \\
\text { protección } \\
\text { de propiedad } \\
\text { intelectual }\end{array}$} & $0,0510^{* *}$ & $2,973^{* * *}$ & $-0,0159$ & $-0,878^{* * *}$ & $-0,00735$ & $-0,357$ & $0,0469^{*}$ & $2,873^{* * *}$ \\
\hline & $-0,024-$ & $-0,528-$ & $-0,025-$ & $-0,245-$ & $-0,022-$ & $-0,267-$ & $-0,027-$ & $-0,807-$ \\
\hline \multirow{2}{*}{$\begin{array}{l}\text { Falta de } \\
\text { protección a la } \\
\text { imitación }\end{array}$} & $-0,0106$ & $-0,463^{* *}$ & $0,0480^{*}$ & $2,129^{* * *}$ & 0,0158 & $0,957^{* * *}$ & 0,0100 & $0,750^{* *}$ \\
\hline & $-0,025-$ & $-0,232-$ & $-0,025-$ & $-0,400-$ & $-0,021-$ & $-0,261-$ & $-0,026-$ & $-0,302-$ \\
\hline \multirow{2}{*}{$\begin{array}{l}\text { Escases de } \\
\text { recursos propios }\end{array}$} & $0,0411^{*}$ & $2,515^{* * *}$ & $0,0610^{* * *}$ & $3,613^{* * *}$ & $0,0483^{* *}$ & $3,030^{* * *}$ & $-0,00229$ & 0,0919 \\
\hline & $-0,023-$ & $-0,437-$ & $-0,024-$ & $-0,628-$ & $-0,020-$ & $-0,384-$ & $-0,025-$ & $-0,245-$ \\
\hline \multirow{2}{*}{$\begin{array}{l}\text { Escases de } \\
\text { información del } \\
\text { mercado }\end{array}$} & $0,0447^{*}$ & $2,225^{* * *}$ & $0,0879^{* * *}$ & $2,405^{* * *}$ & 0,0137 & $0,779^{* * *}$ & 0,0353 & $2,316^{* * *}$ \\
\hline & $-0,023-$ & $-0,483-$ & $-0,023-$ & $-0,456-$ & $-0,021-$ & $-0,253-$ & $-0,025-$ & $-0,646-$ \\
\hline \multirow{2}{*}{$\begin{array}{l}\text { Exportaciones } \\
-\%-\end{array}$} & $0,302^{* * *}$ & $17,25^{* * *}$ & $0,292^{* * *}$ & $13,04^{* * *}$ & 0,00715 & 0,637 & $0,274^{* *}$ & $20,00^{* * *}$ \\
\hline & $-0,057-$ & $-2,670-$ & $-0,063-$ & $-1,880-$ & $-0,083-$ & $-1,001-$ & $-0,126-$ & $-4,439-$ \\
\hline \multirow{2}{*}{$\begin{array}{l}\text { Capital humano } \\
-\% —\end{array}$} & $0,220^{* * *}$ & $14,26^{* * *}$ & $0,160^{* * *}$ & $8,243^{* * *}$ & $0,198^{* * *}$ & $13,45^{* * *}$ & $-0,00609$ & $1,481^{* * *}$ \\
\hline & $-0,044-$ & $-2,062-$ & $-0,047-$ & $-1,140-$ & $-0,028-$ & $-1,312-$ & $-0,034-$ & $-0,321-$ \\
\hline \multirow{2}{*}{$\begin{array}{l}\text { La inversa de la } \\
\text { razón de Mills }\end{array}$} & & $23,49^{* * *}$ & & $18,33^{* * *}$ & & $19,16^{* * *}$ & & $35,73^{* * *}$ \\
\hline & & $-4,220-$ & & $-3,482-$ & & $-2,169-$ & & $-8,725-$ \\
\hline \multirow[t]{2}{*}{ Constante } & & $-36,03^{* * *}$ & & $-23,90^{* * *}$ & & $-32,62^{* * *}$ & & $-39,35^{* * *}$ \\
\hline & & $-6,744-$ & & $-4,896-$ & & $-3,891-$ & & $-10,193-$ \\
\hline Observaciones & 2248 & 2248 & 2254 & 2254 & 2122 & 2122 & 2155 & 2155 \\
\hline
\end{tabular}

Errores estándar están entre guiones. Significancia: ${ }^{*} \mathrm{p}<0,10 ;{ }^{* *} \mathrm{p}<0,05 ;{ }^{* *} \mathrm{p}<0,01$.

Fuente. Elaboración propia con base en DANE, 2017.

Para el segundo periodo de la encuesta en el sector de servicios -2016-2017—, las variables seleccionadas en el vector de características que posibilitan la innovación resultaron significativas, exceptuando la escasez de recursos propios de la firma para emprender una innovación. Adicionalmente, la razón de Mills fue fuertemente significativa para los dos sectores económicos, por lo que considera el sesgo de selección entre las firmas y lo corrige como medida de generación de parámetros consistentes en las estimaciones en ambos sectores.
La estimación de un sesgo de selección entre las observaciones y corregirlo bajo el enfoque de Heckman permite estimar las funciones de producción en las firmas con innovaciones definidas -innovadoras en sentidos estricto y amplio- - y no definidas -innovadoras potenciales - en ambos sectores de la economía colombiana, utilizando la metodología de estimaciones por FMCG. Para esto se tiene como variable dependiente los ingresos corrientes de las firmas y un vector de variables independientes compuestas por el capital físico, 
capital en I+D y trabajo, en términos de logaritmo. De igual forma, se genera un vector de características propias de la firma a fin de identificar los factores que inciden en las mediciones de la productividad (véanse los resultados en la Tabla 3 ).

\subsection{Estimación de la función de producción para firmas innovadoras de servicios y manufactureras}

A continuación, se estima la función de producción para los sectores de servicios e industria manufacturera (véase la Tabla 5). Así, se evidencia que el capital físico y el número de trabajadores son significativos en la estructura de producción de las firmas innovadoras de ambos sectores, lo que reafirma el papel fundamental que tienen estos insumos en la composición económica de las empresas. Por otra parte, las inversiones en $\mathrm{I}+\mathrm{D}$ tienden a ser significativas y positivas en la producción de las empresas del sector manufacturero $y$, parcialmente, en el sector de servicios, mientras que el tamaño de las empresas es significativo para el sector de servicios, pero su efecto es negativo debido a que, en su mayoría, el sector lo componen firmas pequeñas y medianas.

La cooperación con otras instituciones públicas o privadas tiene un efecto negativo en los sectores, de modo que es significativo en el sector de servicios; esta situación implica —en contradicción con la teoría planteada por Agarwal y Selen (2011) sobre la conformación de redes productivas internas y externas de la empresa- que para el caso colombiano una de las debilidades del ecosistema empresarial es la poca representación de la cooperación en el desarrollo de innovaciones.
Uno de los aspectos comunes en las encuestas de servicios y manufacturas es la falta de recursos propios para emprender innovaciones, de manera que se estructura como un factor fundamental en la generación de la producción en las empresas innovadoras y que evidenció impactos negativos. Con respecto al porcentaje de capital humano que se encuentra en las empresas, donde se capta información de personas con formación de posgrado, universitarios, técnicos y tecnólogos y se conforma la razón entre el personal cualificado sobre el total de los empleados, es posible concluir que esta es significativa y positiva a lo largo de la encuesta, al igual que los resultados obtenidos por Pasban y Nojedeh (2016).

En el análisis de coeficientes se obtiene que el término de la constante es significativo para todas las regresiones hechas; por términos de la teoría, si las elasticidades son cero para las variables explicativas, esto genera que los coeficientes de la constante sean interpretados como la PTF o el residuo de Solow, y su análisis muestra la productividad media de las firmas (Sala-i-Martín, 2000). En este sentido, comparando ambos sectores en los dos periodos, el sector de servicios tiende a tener mediciones de productividad mayores en comparación con el sector manufacturero (véase la Tabla 4).

A continuación, se analizan los niveles de rendimientos constantes a escala (RCE) de la producción, que en la suma de las elasticidades es igual a 1 ; en otras palabras, la validación de la hipótesis nula en el test de RCE. Así, evaluando para servicios y la industria manufacturera se evidencia la ausencia de rendimientos constantes a escala, calculados en la tabla 4. 
Tabla 4. Test de RCE para el sector de servicios y manufactura en Colombia

\begin{tabular}{|c|c|c|c|c|}
\hline & EDITM VII & EDITM VIII & EDITS V & EDITS VI \\
\hline Test de RCE & 0,000 & 0,000 & 0,0035 & 0,000 \\
\hline
\end{tabular}

Fuente. Elaboración propia.

Tabla 5. Estimación de función de producción para los sectores manufacturero y servicios en Colombia

\begin{tabular}{|c|c|c|c|c|c|c|c|c|}
\hline & \multicolumn{2}{|c|}{ EDITM 2013-2014 } & \multicolumn{2}{|c|}{ EDITM 2015-2016 } & \multicolumn{2}{|c|}{ EDITS 2014-2015 } & \multicolumn{2}{|c|}{ EDITS 2016-2017 } \\
\hline & Coef & SE & Coef & SE & Coef & SE & Coef & SE \\
\hline $\log$ de $I+D$ & $0,00791^{*}$ & $-0,004-$ & $0,0168^{* * *}$ & $-0,004-$ & $0,0156^{* * *}$ & $-0,005-$ & 0,00369 & $(0,006)$ \\
\hline Log capital físico & $0,0252^{* * *}$ & $-0,004-$ & $0,0174^{* * *}$ & $-0,005-$ & $0,0393^{* * *}$ & $-0,006-$ & $0,0401^{* * *}$ & $(0,007)$ \\
\hline $\begin{array}{l}\text { Log del total de } \\
\text { trabajadores }\end{array}$ & $1,171^{* * *}$ & $-0,021-$ & $1,143^{* * *}$ & $-0,022-$ & $0,920^{* * *}$ & $-0,043-$ & $0,997^{* * *}$ & $(0,042)$ \\
\hline $\begin{array}{l}\text { Tamaño de la } \\
\text { empresa }\end{array}$ & $-0,0297$ & $-0,062-$ & 0,102 & $-0,066-$ & $-0,182^{*}$ & $-0,098-$ & $-0,379^{* * *}$ & $(0,094)$ \\
\hline IED & 0,0393 & $-0,042-$ & $0,0967^{* *}$ & $-0,043-$ & 0,0535 & $-0,065-$ & $0,226^{* * *}$ & $(0,064)$ \\
\hline Cooperación & $-0,0218$ & $-0,035-$ & $-0,0193$ & $-0,037-$ & $-0,271^{* * *}$ & $-0,058-$ & $-0,0867$ & $(0,057)$ \\
\hline $\begin{array}{l}\text { Falta de personal } \\
\text { cualificado }\end{array}$ & $-0,0618$ & $-0,038-$ & $-0,0550$ & $-0,038-$ & $-0,0606$ & $-0,064-$ & $-0,0579$ & $(0,059)$ \\
\hline $\begin{array}{l}\text { Falta de protección } \\
\text { de propiedad } \\
\text { intelectual }\end{array}$ & 0,0515 & $-0,040-$ & $-0,00778$ & $-0,040-$ & $-0,0366$ & $-0,066-$ & 0,0177 & $(0,069)$ \\
\hline $\begin{array}{l}\text { Falta de protección } \\
\text { a la imitación }\end{array}$ & $-0,118^{* * *}$ & $-0,041-$ & $-0,0185$ & $-0,042-$ & $-0,0197$ & $-0,062-$ & $-0,0473$ & $(0,063)$ \\
\hline $\begin{array}{l}\text { Escases de } \\
\text { recursos propios }\end{array}$ & $-0,149^{* * *}$ & $-0,039-$ & $-0,167^{* * *}$ & $-0,041-$ & $-0,323^{* * *}$ & $-0,067-$ & $-0,269^{* * *}$ & $(0,061)$ \\
\hline $\begin{array}{l}\text { Escases de } \\
\text { información del } \\
\text { mercado }\end{array}$ & 0,0567 & $-0,037-$ & $-0,0518$ & $-0,039-$ & $-0,0977$ & $-0,064-$ & $-0,0489$ & $(0,059)$ \\
\hline $\begin{array}{l}\text { Exportaciones } \\
-\%-\end{array}$ & $0,414^{* * *}$ & $-0,113-$ & $0,398^{* * *}$ & $-0,130-$ & $1,420^{* * *}$ & $-0,342-$ & $1,513^{* * *}$ & $(0,355)$ \\
\hline $\begin{array}{l}\text { Capital humano } \\
-\%-\end{array}$ & $1,339^{* * *}$ & $-0,094-$ & $1,352^{* * *}$ & $-0,102-$ & $0,452^{* * *}$ & $-0,107-$ & $0,416^{* * *}$ & $(0,092)$ \\
\hline Constante & $9,598^{* * *}$ & $-0,099-$ & $10,43^{* * *}$ & $-0,101-$ & $11,83^{* * *}$ & $-0,193-$ & $11,53^{* * *}$ & $(0,198)$ \\
\hline Observaciones & 2248 & & 2254 & & 2122 & & 2155 & \\
\hline$R$ cuadrado & 0,8370 & & 0,8200 & & 0,6160 & & 0,6176 & \\
\hline $\begin{array}{l}\mathrm{R} \text { cuadrado } \\
\text { ajustado }\end{array}$ & 0,8361 & & 0,8190 & & 0,6136 & & 0,6153 & \\
\hline
\end{tabular}

Errores estándar están entre guiones. Significancia: ${ }^{*} \mathrm{p}<0,10 ;{ }^{* *} \mathrm{p}<0,05 ;{ }^{* *} \mathrm{p}<0,01$.

Fuente. Elaboración propia con base en DANE, 2017. 
Calculando la productividad total de los factores o el residuo de Solow y al examinar la homogeneidad de productividades de los sectores de servicios y manufacturas por medio de análisis de densidad, se identifica que la homogeneidad de productividades de las empresas innovadoras pertenecientes al sector manufacturero en Colombia obtiene una menor dispersión. No obstante, para las empresas innovadoras - de innovación definida como no definida - del sector de servicios es posible identificar que se caracterizan por su mayor dispersión de las productividades, con mediciones más heterogéneas.

Figura 2. Análisis de densidad de productividad para el sector de servicios y manufactura en Colombia

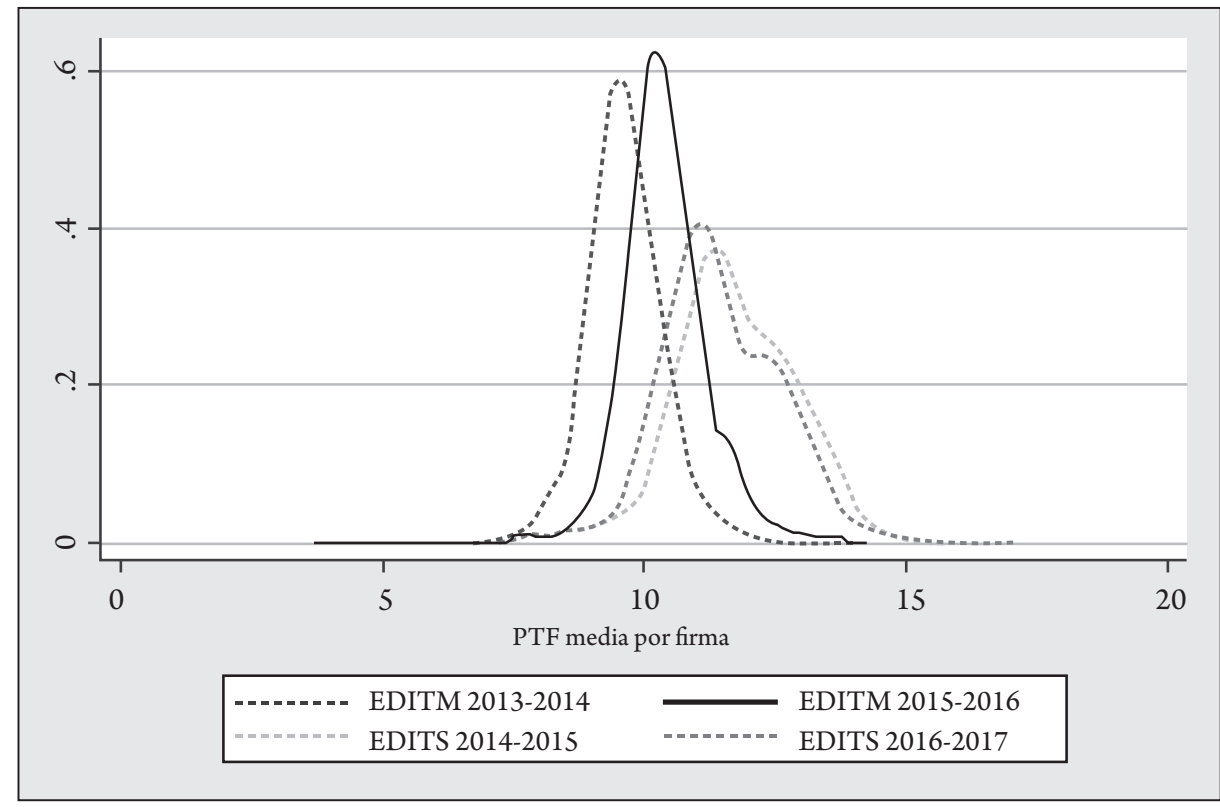

Fuente. Elaboración propia. 


\section{Conclusiones}

$\mathrm{A}^{\mathrm{n}}$ nalizar los factores que impulsan la propensión a innovar y el desempeño de la productividad en los sectores de servicios e industria manufacturera en Colombia fue el objetivo de este artículo, para lo cual se utilizaron los datos de las Encuestas de Innovación y Desarrollo Tecnológico para ambos sectores; al calcular la propensión a innovar y la intensidad de la innovación con base en la inversión en I+D, mediante el enfoque de dos etapas, se identificó que el tamaño de las empresas, la cooperación con instituciones privadas o públicas, el porcentaje de exportaciones y de capital humano resultaron significativos y positivos para el desarrollo de innovaciones. La presencia de IED es significativa, pero con una relación negativa para la propensión a innovar.

Los principales insumos de producción, tales como la acumulación de capital, el trabajo y el capital en I+D son significativos y resultaron positivos al estar acordes con la concepción de la teoría económica. Aspectos relacionados con el tamaño de las empresas son significativos en el sector de servicios, pero no para el sector manufacturero, situación que puede explicarse por la composición sectorial de los servicios que está conformado, en mayor medida, por medianas y pequeñas empresas. De igual forma, el efecto de la IED y de la cooperación con otras instituciones tiende a ser variante a través de los sectores, valorándose su significancia más en el sector de servicios.

En relación con las barreras que presentan las firmas, un aspecto generalizado es la ausencia de recursos que tienen las empresas para financiarse y generar nuevos proyectos de innovación, lo cual resultó significativo en la disminución del valor agregado de las firmas. Aspectos tales como el porcentaje de exportaciones y el capital humano empleado generan un efecto positivo en la concepción de la producción de las empresas innovadoras en el sector de servicios y manufacturas.
La constante de cada modelo es significativa y positiva, de modo que se interpreta como la PTF media real, siendo mucho mayor para el sector de servicios debido a su vocación tecnológica. Ahora bien, las empresas innovadoras no poseen rendimientos constantes a escala (RCE) debido a la existencia de un efecto multiplicador por la concepción de la innovación.

En el cálculo de la PTF y la cuantificación de la densidad de productividad, las empresas del sector manufacturero poseen niveles más homogéneos en ambos periodos, localizándose la mayor parte de las industrias en el punto central; así mismo, las empresas del sector de servicios ostentan productividades más heterogéneas.

La evidencia indica que la media de las empresas del sector de manufactura tiene una productividad proporcional o convergente; en el caso de los servicios que son intensivos en mano de obra y en conocimientos acumulados, las discrepancias de la mano de obra en cada subsector llevan a que exista una productividad alejada de su media.

Finalmente, fue posible evidenciar qué insumos básicos destinados a actividades de desarrollo de conocimiento poseen un efecto positivo en los ingresos de las empresas, de modo que se hace necesario fortalecer las redes de formación y utilización de insumos en la cadena de producción. Es fundamental, entonces, que las empresas, dentro del entramado productivo, posean ecosistemas con mayor cooperación entre entidades públicas y privadas que estimulen el crecimiento de la productividad y de los ingresos percibidos.

Además, resulta evidente la necesidad de un conjunto de políticas que mejoren la propensión a innovar de las empresas colombianas y estimulen la productividad por medio de la mejora de los encadenamientos, las capacidades de competir en mercados internacionales 
y la inclusión de talento humano con capacidades dinámicas que fortalezcan la cultura de la innovación, las capacidades de absorción tecnológica y de utilización eficiente del conocimiento.

\section{Referencias}

Aboal, D.; Tacsir, E. (2018). Innovation and productivity in services and manufacturing: the role of ICT. Industrial and Corporate Change, 27(2), 221-241. DOI: https://doi. org/10.1093/icc/dtx030

Agarwal, R.; Selen, W. (2011). Multi-dimensional nature of service innovation: operationalisation of the elevated service offerings construct in collaborative. International Journal of Operations \& Production Management , 31(11), 1164-1192. DOI: https://doi.org/10.1108/01443571111178484

Beveren, I. van. (2012). Total factor productivity estimation: a practical review. Journal of Economic Surveys, 26(1), 98-128. DOI: https://doi.org/10.1111/j.1467-6419.2010.00631.x

Broadberry, S. N. (1995). Comparative industrial productivity levels in manufacturing since the Revolution: lessons from Britain, America, Germany and Japan. Structural Change and Economic Dynamics, 6(1), 71-95. DOI: https://doi. org/10.1016/0954-349X(94)00004-S

Brondino, G. (2019). Productivity growth and structural change in China (1995-2009): a subsystems analysis. Structural Change and Economic Dynamics, 49(C),183-191. DOI: https://doi.org/10.1016/j.strueco.2018.09.001

Cainelli, G.; Evangelista, R.; Savona, M. (2006). Innovation and economic performance in services : a firm-level analysis. Cambridge Journal of Economics, 30(3), 435-458. DOI: https://doi.org/10.1093/cje/bei067

Camino-Mogro, S. (2017). Estimación de una función de producción y análisis de la productividad: el sector de innovación global en mercados locales. Estudios Gerenciales, 33(145), 400-411. DOI: https://doi.org/10.1016/j. estger.2017.10.004

Carvalho, L.; Avellar, A. (2017). Innovation and productivity: empirical evidence for Brazilian industrial enterprises. Revista de Administração, 52(2), 134-147. DOI: https://doi. org/10.1016/j.rausp.2016.12.009

Chapman, R.; Soosay, C.; Kandampully, J. (2002). Innovation in logistic services and the new business model : a conceptual framework. Managing Services Quality, 12(6), 358-371. DOI: https://doi.org/10.1108/09604520210451849
Chen, S.; Jefferson, G. H.; Zhang, J. (2011). China Economic Review structural change, productivity growth and industrial transformation in China. China Economic Review, 22(1), 133150. DOI: https://doi.org/10.1016/j.chieco.2010.10.003

Chung, H. (2018). ICT investment-specific technological change and productivity growth in Korea : comparison of 1996-2005 and 2006-2015. Telecommunications Policy, 42(1), 78-90. DOI: https://doi.org/10.1016/j.telpol.2017.08.005

Crespi, G.; Tacsir, E.; Vargas, F. (2016). Innovation dynamics and productivity: evidence for Latin America. En M. Grazzi; P. Carlo (Eds.) Firm innovation and productivity in Latin America and the Caribbean: the engine of economic development. (37-72). Washington D. C.: Inter-American Development Bank.

Crespi, G.; Zuniga, P. (2012). Innovation and productivity: evidence from six Latin American countries. World Development, 40(2), 273-290. DOI: https://doi. org/10.1016/j.worlddev.2011.07.010

DANE. (2017). Encuesta de desarrollo e innovación tecnológica (EDIT). Recuperado de https://bit.ly/2J2B63v

Dimelis, S. P.; Papaioannou, S. K. (2011). ICT growth effects at the industry level : a comparison between the US and the EU. Information Economics and Policy, 23(1), 37-50. DOI: https:// doi.org/10.1016/j.infoecopol.2010.03.004

Eslava, M.; Haltiwanger, J.; Kugler, A.; Kugler, M. (2013). Trade and market selection: evidence from manufacturing plants in Colombia. Review of Economic Dynamics, 16(1), 135-158. DOI: https://doi.org/10.1016/j.red.2012.10.009

Fleury, A. (1995). Quality and productivity in the competitive strategies of Brazilian industrial enterprises. World Development, 23(1), 73-85. DOI: https://doi. org/10.1016/0305-750X(94)00113-D

Grönroos, C.; Ojasalo, K. (2015). Service productivity as mutual learning. International Journal of Quality and Service Sciences, 7(2/3), 296-311. DOI: https://doi.org/10.1108/ IJQSS-03-2015-0035

Hall, B. H.; Lotti, F.; Mairesse, J. (2009). Innovation and productivity in SMEs : empirical evidence for Italy. Small Business Economics, 33, 13-33. DOI: https://doi.org/10.1007/ s11187-009-9184-8 
Halpern, L.; Muraközy, B. (2012). Innovation, productivity and exports : the case of Hungary. Economics of Innovation and New Technology, 21(2), 151-173. DOI: https://doi.org/10.1080/1 0438599.2011 .561995

Haskel, J.; Wallis, G. (2013). Public support for innovation, intangible investment and productivity growth in the UK market sector. Economics Letters, 119(2), 195-198. DOI: https://doi.org/10.1016/j.econlet.2013.02.011

Heckman, J. (1976). The common structure of statitical models of truncation, sample selection and limited dependent variable and simpler estimation and such models. En B. Stanford (Ed.) The Annals of Economic and Social Measurenment, Vol. 5, Núm 4. (475-492). Cambridge, MA: NBER

Heggedal, T.; Moen, E. R.; Preugschat, E. (2017). Productivity spillovers through labor mobility in search. Journal of Economic Theory, 169, 551-602. DOI: https://doi.org/10.1016/j. jet.2017.03.003

Hussinger, K. (2008). R \& D and subsidies at the firm level: an application of parametric and semiparametric two-step selection models. Journal of Applied Econometrics, 23(6), 729 747. DOI: https://doi.org/10.1002/jae.1016

Junarsin, E. (2010). Issues in the innovation service product process : a managerial perspective. International Journal of Management, 27(3).

Kleinknecht, A. (1996). New indicators and determinantes of innovation: an introduction. En A. Kleinknecht (Ed.) Determinants of innovation. (1-13). Londres: MacMillan Press. DOI: https://doi.org/10.1007/978-1-349-13917-0

Klomp, L.; Leeuwen, G. van. (2001). Linking innovation and firm performance: a new approach. International Journal of the Economics of Business, 8(3), 343-364. DOI: https://doi. org/10.1080/13571510110079612

Kriaa, M.; Karray, Z. (2010). Innovation and R\&D investment of Tunisian firms: a two-regime model with selectivity correction. The Journal of Business Inquiry, 9(1), 1-21.

Lee, R.; Lee, J.; Garrett, T. C. (2019). Synergy effects of innovation on firm performance. Journal of Business Research, 99, 507515. DOI: https://doi.org/10.1016/j.jbusres.2017.08.032

Levinsohn, J.; Petrin, A. (2003). Estimating production functions using inputs to control for unobservables. The Review of Economic Studies. 70(2), 317-341. DOI: https://doi. org/10.1111/1467-937X.00246

Machado, R.; Tello-Gamarra, J. (2017). Inovação em serviços: estado da arte e perspectivas futuras. Suma de Negocios, 8(17), 1-10. DOI: https://doi.org/10.1016/j.sumneg.2017.01.002

Máñez, J.; Rochina-Barrachina, M.; Sanchis, A.; Sanchis, J. A. (2013). Do process innovations boost SMEs productivity growth? Empirical Economics, 44(3), 1373-1405. DOI: https://doi.org/10.1007/s00181-012-0571-7
Messa, A. (2014). Metodologias de cálculo da produtividade total dos fatores e da produtividade da mão de obra. En F. de Negri; L. Calvacante (Orgs.) Productividade no Brasil: desempenho e determinantes. (87-109). IPEA.

Mestieri, M.; Schauer, J.; Townsend, R. M. (2017). Human capital acquisition and occupational choice : implications for economic development. Review of Economic Dynamics, 25, 151-186. DOI: https://doi.org/10.1016/j.red.2017.02.001

Montes-Rojas, G.; Santamaría, M. (2007). Sources of productivity growth : evidence from the Mexican manufacturing sector. The North American Journal of Economics and Finance, 18(3), 263-278. DOI: https://doi.org/10.1016/j.najef.2007.06.004

Morikawa, M. (2019). Innovation in the service sector and the role of patents and trade secrets: evidence from Japanese firms. Journal of The Japanese and International Economies, 51, 43-51. DOI: https://doi.org/10.1016/j.jjie.2018.10.003

Nakamura, K.; Kaihatsu, S.; Yagi, T. (2019). Productivity improvement and economic growth: lessons. Economic Analysis and Policy, 62, 57-79.DOI: https://doi.org/10.1016/j. eap.2018.11.002

OECD; Eurostat. (2018). Oslo Manual, guidelines for collecting, reporting and using data on innovation (4a ed.). París: OECD Publishing. Recuperado de https://bit.ly/3nZOiVz

Ozyilmaz, A. (2001). Service innovation audit and role of information technology in service innovation. Nueva York: Rensselaer Polytechnic Institute.

Pasban, M.; Nojedeh, S. H. (2016). A review of the role of human capital in the organization. Procedia-Social and Behavioral Sciences, 230, 249-253. DOI: https://doi.org/10.1016/j. sbspro.2016.09.032

Petrin, A.; Poi, B.; Levinsohn, J. (2004). Production function estimation in Stata using inputs to control for unobservables. The Stata Journal: Promoting communications on statistics and Stata 4(2), 113-123. DOI: https://doi. org/10.1177/1536867X0400400202

Ramírez, J. M.; Zubieta, I.; Bedoya, J. G. (Agosto de 2014). Productividad y competitividad del sistema de ciudades. Fedesarrollo. Bogotá, Colombia. Recuperado de https://bit. ly/3fBLwmt

Rath, B. N. (2018). Productivity growth and efficiency change : comparing manufacturing and service-based firms in India. Economic Modelling, 70, 447-457. DOI: https://doi. org/10.1016/j.econmod.2017.08.024

Raymond, L.; St-Pierre, J. (2010). R \& D as a determinant of innovation in manufacturing SMEs : an attempt at empirical clarification. Technovation, 30(1), 48-56. DOI: https://doi. org/10.1016/j.technovation.2009.05.005

Sala-i-Martín, X. (2000). Apuntes de crecimiento económico (2a ed.). España: Antoni Bosch. 
Sánchez-Sellero, P.; Sánchez-Sellero, M. C.; Sánchez-Sellero, F. J.; Cruz-González, M. M. (2014). Innovación y productividad manufacturera. Journal of Technology Management and Innovation, 9(3), 135-145. DOI: https://doi.org/10.4067/ S0718-27242014000300010

Singh, G.; Gupta, A.; Juneja, C. (2018). Productivity measurement of manufacturing system. Materials Today: Proceedings, 5(1), 1483-489. DOI: https://doi.org/10.1016/j. matpr.2017.11.237

Subramanian, U.; Anderson, W. P.; Lee, K. (2005). Measuring the impact of the investment climate on total factor productivity: the cases of China and Brazil (N.o 3792). World Bank, Washington, DC., EE.UU. DOI: https://doi.org/10.1596/1813-94503792

Svyerson, C. (2011). What determines productivity? Journal of Economic Literature, 49(2), 326-365. DOI: https://doi. org/10.1257/jel.49.2.326

Tello, M. D. (2017). Innovación y productividad en las empresas de servicios y manufactureras: el caso del Perú. Cepal Review, (121), 73-92. DOI: https://doi.org/10.18356/78513868-es

Van Biesebroeck, J.(2004). Robustness of productivity estimates. The Journal of Industrial Economics, 55(3), 529-569. DOI: https://doi.org/10.1111/j.1467-6451.2007.00322.x

Vang, J.; Zellner, C. (2005). Introduction : innovation in services. Industry and Innovation, 12(2), 147-152. DOI: https://doi. org/10.1080/13662710500087875

Varella, A.; Cabral, R. (2009). Productivity effects on Mexican manufacturing employment. The North American Journal of Economics and Finance, 20(1), 66-81. DOI: https://doi. org/10.1016/j.najef.2008.10.002

Veugelers, R. (1997). Internal R\&D expenditures and external technology sourcing. Research Policy, 26(3), 303-315. DOI: https://doi.org/10.1016/S0048-7333(97)00019-X

Villarreal, N. F.; Lucio-Arias, D.; Albis, N.; Mora, H. (2014). Determinantes de la innovación y la productividad en la industria manufacturera colombiana por tamaño de firma. Bogotá, Colombia. Recuperado de https://bit.ly/2V4eB0a

Weerawardena, J.; Mccoll-kennedy, J. R. (2002). New service development and competitive advantage: a conceptual model. Australasian Marketing Journal, 10(1), 13-23. DOI: https://doi.org/10.1016/S1441-3582(02)70140-7

Weinstein, O.; Gallouj, F. (1997). Innovation in services. Research Policy, 26(4-5), 537-556. DOI: https://doi.org/10.1016/ S0048-7333(97)00030-9

Zagler, M. (2002). Services, innovation and the new economy. Structural Change and Economic Dynamics, 13(3), 337-355. DOI: https://doi.org/10.1016/S0954-349X(02)00004-8 